\title{
Photonic information processing needs quantum design rules
}

\author{
Neil Gunther, Edoardo Charbon, Dmitri Boiko, and Giordano \\ Beretta
}

The quantum nature of light requires engineers to have a special set of design rules for fabricating photonic information processors that operate correctly.

In the century since Einstein postulated light's quantum nature-an event celebrated last year, along with SPIE's 50th birthday-the quantum revolution has progressed from formalizing the mathematical theory of the electron and photon, to a second wave that uses quantum phenomena for new information-processing technologies. These applications are found in fields as diverse as communications, cryptography, computing, and even imaging.

Since photons are the de facto information-encoding entity, this means photonic information processing often amounts to quantum information processing, which can be carried out on chips fabricated with very large scale integration (VLSI) techniques, such as the array of single-photon detectors shown in Figure 1, for significant cost reduction. Our experience with VLSI has taught us the importance of having good rules $^{1}$ that abstract away the underlying physics and allow engineers to design chips. This inspired us to consider an analogous set of quantum design rules (QDRs) for VLSI photonics., ${ }^{2,3}$

Understanding the quantum rules

To establish correct engineering guidelines, we first need to understand the quantum rules that photons obey. We adopt the quantum path integral (QPI) formalism ${ }^{4}$ to avoid traps that arise from treating the photon as either a classical particle or a classical wave. Under this formalism, a photon propagating in free space is represented by an infinite set of QPI amplitudes $\phi_{i}$ between source $s$ and detector $d$.

The probability for detecting a physical photon is determined by summing over all of these paths, and then taking the squared modulus of the result. Some eight quantum rules ${ }^{2,3}$ of this type will correctly calculate probabilities in VLSI informationprocessing devices. For example, in Figure 2, which shows a scat-

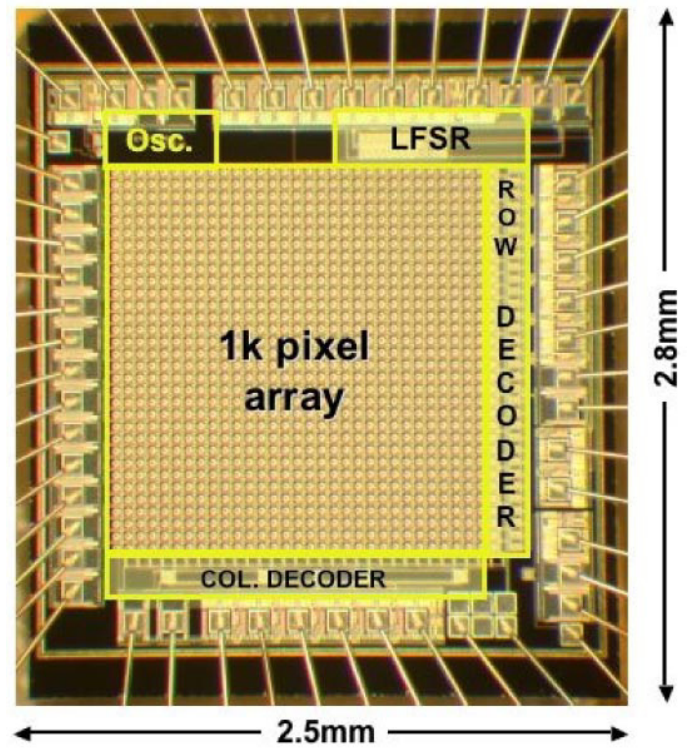

Figure 1. This device includes a $32 \times 32$ array of CMOS single-photon detectors.

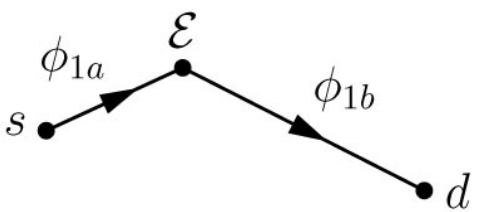

Figure 2. Shown is one of infinitely many paths $\phi$ a photon can take going from source s to detector $\mathrm{d}$. Path $\phi_{1}$ includes an interaction with matter, an event denoted by $\mathcal{E}$, and two legs, $\phi_{1 a}$ and $\phi_{1 b}$.

tering event $\mathcal{E}$, path $\phi_{1}$ would be computed as the product of the two legs, $\phi_{1 a}$ and $\phi_{1 b}$, an example of the 'AND-ing' rule. We have shown elsewhere that flawed claims can result when these rules are ignored. ${ }^{2}$

Continued on next page 


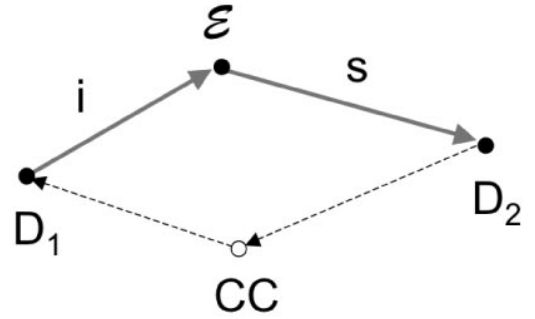

Figure 3. The entangled biphoton may be viewed as a quantum path integral amplitude with idler and signal segments, $\mathrm{i}$ and s, created by event $\mathcal{E} . D_{1}, D_{2}$ : Detectors. CC: Coincidence counter.

\section{Entanglement, loops, and ghosts}

We have extended QPI formalism to entanglement., ${ }^{2,3}$ The most common method for producing entangled 'biphotons' pumps a nonlinear crystal, such as beta-barium borate (BBO), with UV photons to create photon pairs whose total momenta and energy equal that of each pump photon. An entangled pair acts like the two-segment QPI amplitude shown in Figure 2. Figure 3 depicts the $\mathrm{BBO}$ crystal as the scattering event $\mathcal{E}$ in Figure 2, and detectors $D_{1}$ and $D_{2}$ as the source and detector, respectively. One biphoton (signal) propagates from $\mathcal{E}$ to detector $D_{2}$ in the expected way, but the idler photon propagates backward from $D_{1}$ to $\mathcal{E}$. The coincidence counter (CC) forms a closed circuit of paths.

Figure 3, as adapted in Figure 4, also explains how quantum ghost imaging 5,6 works. Detector $D_{1}$ acts like a source for the idler photon passing through a focusing lens to the BBO crystal, which propagates the signal photon to the detector array $D_{2}$. The ghost image appears only in the coincidence counts, focused in accordance with the thin lens equation.

\section{Toward QDRs}

With the photon rules in hand, we can discern some engineeringlevel QDRs. (i) Photons don't interact with photons, but only the material comprising the device. (ii) The minutest change to the material comprising a quantum information device can completely alter its operation. (iii) The distinction between amplitudes and intensities made in Fourier optics becomes redundant: only quantum amplitudes lead to probabilities. (iv) The thin lens equation $d_{i}^{-1}+d_{o}^{-1}=f^{-1}$ still holds for quantum-ghost imaging devices. ${ }^{5}$ More experiments are needed to enumerate other QDRs, and that is a major thrust of our current research conducted in the Quantum Architecture Group at the Swiss Federal Institute of Technology Lausanne (EPFL; Figure 5).

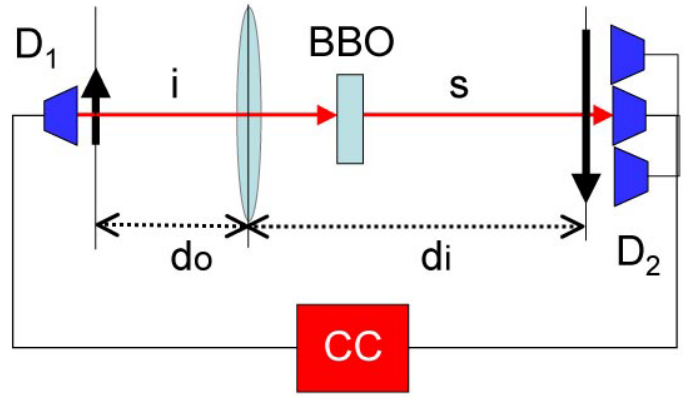

Figure 4. This quantum ghost imaging device is topologically equivalent to the closed-circuit arrangement of Figure 3 with an interposing thin lens. $d_{o}, d_{i}$ : Object and image distances.

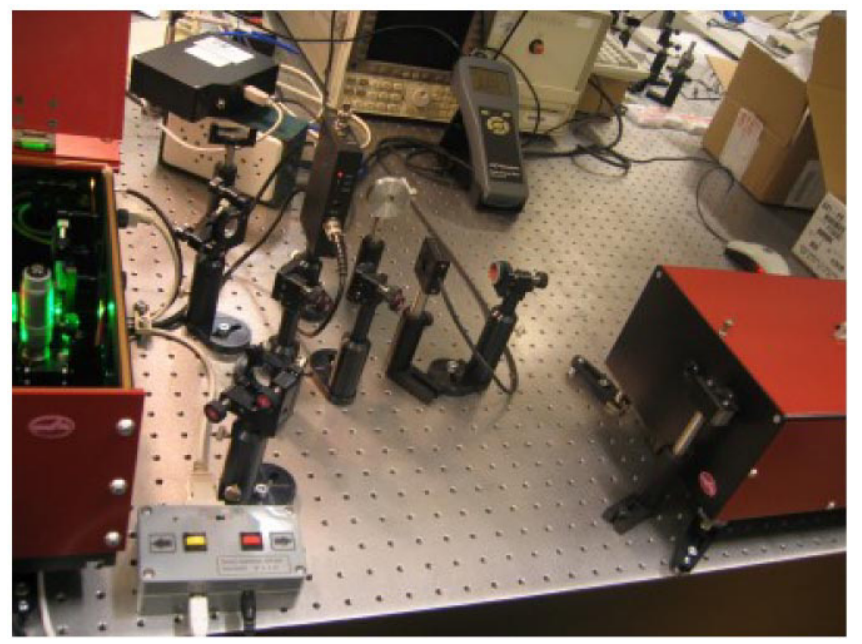

Figure 5. The Quantum Architecture Group at the Swiss Federal Institute of Technology Lausanne uses this laser system to create entangled biphotons.

\section{Author Information}

\section{Neil Gunther}

Performance Dynamics Company

Castro Valley, CA

http:/ / www.perfdynamics.com

Neil Gunther is an internationally recognized consultant who founded Performance Dynamics in 1994. He holds an MSc in applied mathematics and a PhD in theoretical physics. He presented at the SPIE Quantum Communications and Quantum Imaging conferences in 2005 and 2006. 


\section{Edoardo Charbon and Dmitri Boiko}

Faculté Informatique et Communications

EPFL

Lausanne, Switzerland

Edoardo Charbon holds a diploma from the Swiss Federal Institute of Technology Zurich (ETH Zurich), an MS from the University of California, San Diego, and a PhD in electrical engineering and computer sciences from the University of California, Berkeley. He is on the faculty of EPFL and founded its Quantum Architecture Group. He is a member of the technical committees for VLSI-SOC and ESSCIRC.

Dmitri Boiko received MSc and PhD degrees from the Moscow Institute of Physics and Technology while developing the first solid-state chip-laser gyro. He is a scientific consultant to EPFL's Quantum Architecture Group.

\section{Giordano Beretta}

Digital Printing and Imaging Laboratory

Hewlett-Packard Corp.

Palo Alto, CA

Giordano Beretta works in HP's Digital Printing and Imaging Laboratory. He obtained a diploma in mathematics and a PhD in computer science from ETH Zurich, and holds a dozen patents related to color imaging technology. Beretta is a SPIE Fellow.

\section{References}

1. C. Mead and L. Conway, Introduction to VLSI Systems, Addison-Wesley, Reading Mass., 1980

2. N. J. Gunther and G. B. Beretta, Towards practical design rules for quantum communications and quantum imaging devices, Proc. SPIE 5893, pp. 5893-5832, 2005.

3. E. Charbon, N. J. Gunther, D. L. Boiko, and G. B. Beretta, Design rules for quantum imaging devices: experimental progress using CMOS single photon detectors, Proc. SPIE 6305, pp. 6305-6323, 2006.

4. R. P. Feynman, QED: The Strange Theory of Light and Matter, Alix G. Mautner Memorial Lectures, Princeton University Press, Princeton, N.J., 1985.

5. M. D'Angelo and Y. Shih, Quantum imaging, Laser Physics special issue, 2005.

6. R. E. Meyers and K. S. Deacon, Quantum ghost imaging experiments, Proc. SPIE 6305, pp. 6305-6324, 2006. 